Background Systemic lupus erythematosus (SLE) is a chronic multiorgan autoimmune disease with high morbidity. The pathogenesis is multifactorial. Gut dysbiosis plays a role in the pathogenesis of systemic lupus erythematosus (SLE). It can cause systemic inflammation.

Methods We conducted a randomized, double-blind, placebocontrolled trial to investigate whether synbiotic supplementation could improve gut microbiota composition and function in patients with SLE.

Results Forty six SLE patients were randomised to two groups: the synbiotic group received synbiotic capsule (Lactobacillus helveticus R0052 60\%, Bifidobacterium infantis R0033 20\%, Bifidobacterium bifidum R0071 20\% and $80 \mathrm{mg}$ fructo-oligosaccharides) for 60 days and placebo group. In the synbiotic-supplemented group, 21 patients completed the intervention. In the placebo group, one was excluded from the final analysis because she needed antibiotic treatment for 33 days. We analysed 16s rRNA microbiome data from 89 faecal samples of the 46 patients. We found increases in the Firmicutes to Bacteroidetes ratio, butyrate metabolism and nitrotoluene degradation after synbiotic supplementation. We also found a decrease in potentially pathogenic species and an increase in beneficial species.

Conclusion Synbiotic supplementation affect the composition and functions of gut microbiota in patients with systemic lupus erythematosus.

\section{BARICITINIB-INDUCED CHANGES IN STAT-ASSOCIATED GENE EXPRESSION IN SYSTEMIC LUPUS ERYTHEMATOSUS}

${ }^{1}$ Thomas Dörner, ${ }^{2}$ Yoshiya Tanaka, ${ }^{3}$ Michelle Petri, ${ }^{4}$ Josef S Smolen, ${ }^{5}$ Daniel J Wallace, ${ }^{6}$ Ernst R Dow, ${ }^{6}$ Damiano Fantini, ${ }^{6}$ Richard E Higgs, ${ }^{6}$ Guilherme Rocha, ${ }^{6}$ Brenda Crowe, ${ }^{6}$ Robert J Benschop, ${ }^{6}$ Nicole L Byers, ${ }^{6}$ Maria E Silk, ${ }^{6}$ Stephanie de Bono, ${ }^{6}$ Robert W Hoffman. ${ }^{1}$ Charite Universitätsmedizin Berlin and Deutsches Rheumaforschungszentrum (DRFZ), Berlin, Germany; ${ }^{2}$ University of Occupational and Environmental Health Japan, Kitakyushu, Japan; ${ }^{3}$ Johns Hopkins University School of Medicine, Baltimore, USA; ${ }^{4}$ Medical University of Vienna, Vienna, Austria; ${ }^{5}$ Cedars-Sinai Medical Center/University California at Los Angeles, Los Angeles; ${ }^{6}$ Eli Lilly and Company, Indianapolis, USA

\subsection{6/lupus-2020-eurolupus. 18}

Background Baricitinib, an oral selective Janus kinase (JAK)1 and 2 inhibitor, resulted in significant clinical improvements in patients with active systemic lupus erythematosus (SLE) receiving standard background therapy in the phase 2 study JAHH (NCT02708095). Baricitinib may impact key cytokines implicated in the pathogenesis of SLE through effects on the JAK/ signal transducer and activator of transcription (STAT) signaling pathway. The impact of baricitinib on STAT-related gene expression and associations with clinical response in SLE were evaluated.

Methods 314 patients were randomized 1:1:1 to receive oncedaily placebo, baricitinib 2-mg, or baricitinib 4-mg for 24 weeks in JAHH. Patients were $\geq 18$ years of age, had a

Abstract 04 Table 1 Number of STAT -related genes whose variation has statistically significant association to SLEDAI-2K variation

\begin{tabular}{lcccc}
\hline STAT & Week & $\begin{array}{c}\text { Number of } \\
\text { downstream genes }\end{array}$ & $\begin{array}{c}\text { Number of } \\
\text { downstream genes } \\
\text { with FDR }<0.1\end{array}$ & $\begin{array}{c}\text { Number of } \\
\text { downstream genes } \\
\text { with P }<0.05\end{array}$ \\
\hline STAT1 & 2 & 135 & 30 & 45 \\
STAT1 & 4 & 135 & 0 & 5 \\
STAT1 & 12 & 135 & 0 & 2 \\
STAT1 & 24 & 135 & 0 & 1 \\
STAT2 & 2 & 38 & 11 & 13 \\
STAT2 & 4 & 38 & 0 & 2 \\
STAT2 & 12 & 38 & 0 & 0 \\
STAT2 & 24 & 38 & 0 & 0 \\
STAT4 & 2 & 6 & 0 & 1 \\
STAT4 & 4 & 6 & 0 & 0 \\
STAT4 & 12 & 6 & 0 & 0 \\
STAT4 & 24 & 6 & 0 & 0 \\
\hline
\end{tabular}

Spearmen rank coefficient correlations between variation in SLEDAI-2K (from baseline) and variation in log2 expression (from baseline) of genes were computed at weeks 2, 4, 12, and 24 with baricitinib 4-mg treatment and tested for statistically significant differences from zero. Genes analyzed were downstream of STAT1, STAT2, or STAT4 as selected from the MetaCore Database. The number of selected downstream genes for each STAT is shown, along with the number of statistically significant genes. FDR=false discovery rate; SLEDAI-2K=Systemic Lupus Erythematosus Disease Activity Index-2000; STAT=signal transducer and activator of transcription 
diagnosis of SLE, and had active disease involving skin or joints. RNA isolated from whole blood at baseline and weeks 2, 4, 12, and 24 was analyzed using Affymetrix HTA2.0 array.

Results Gene expression profiling demonstrated a statistically significant elevation of STAT1 and STAT2 gene expression at baseline in SLE patients. There was a significant association between the overexpression of STAT1 and STAT2 at baseline. Baricitinib 4-mg treatment resulted in modest reduction in STAT1, STAT2, and STAT4 expression, and a statistically significant reduction in multiple genes downstream of STAT1, STAT2, and STAT4. The reduction in expression of STAT-associated genes with baricitinib treatment correlated with clinical improvement in SLE patients using SLEDAI-2K measurements (table 1).

Conclusions Baricitinib may partially mediate its effect in SLE through changes in STAT-related gene expression, with changes associated with clinical improvement in SLE.

Acknowledgements Funded by Eli Lilly and Company.

\section{O5 WORLDWIDE TRENDS IN ALL-CAUSE MORTALITY OF AUTO-IMMUNE SYSTEMIC DISEASES BETWEEN 2001 AND 2014}

\begin{abstract}
1,2,3 Marc Scherlinger, ${ }^{3,4}$ Philippe Mert, ${ }^{3,4}$ Flora Sagez, ${ }^{3,4}$ Alain Meyer, ${ }^{3,4}$ Renaud Felten, ${ }^{3,4}$ Emmanuel Chatelus, ${ }^{3,4}$ Rose-Marie Javier, ${ }^{3,4}$ Christelle Sordet, ${ }^{3,5,6}$ Thierry Martin, ${ }^{3,5,6}$ Anne-Sophie Korganow, ${ }^{3,5,6}$ Aurélien Guffroy, ${ }^{3,5}$ Vincent Poindron, 1,2,3 Christophe Richez, 1,2,3 Marie-Elise Truchetet, 2,3Patrick Blanco, 1,2 Thierry Schaeverbeke, ${ }^{2,3}$ Jean Sibilia, ${ }^{7,8}$ Hervé Devillers, ${ }^{3,4,6}$ Laurent Arnaud. ${ }^{1}$ Centre Hospitalier Universitaire de Bordeaux, FHU ACRONIM, Bordeaux; ${ }^{2}$ CNRS-UMR 5164 ImmunoConcEpT, Bordeaux; ${ }^{3}$ Centre National de Référence des maladies auto-immunes et systémiques rares Est/SudOuest (RESO); ${ }^{4}$ Service de rhumatologie, Centre Hospitalier Universitaire de Strasbourg Strasbourg; ${ }^{5}$ Service d'immunologie clinique, Centre Hospitalier Universitaire de Strasbourg, Strasbourg; ' Immuno-rhumatologie moléculaire, INSERM UMR-S 1109, Strasbourg; ${ }^{7}$ Service de Médecine Interne et Maladies Systémiques (médecine interne 2), Centre Hospitalier Universitaire de Dijon, Hôpital François-Mitterrand, Dijon; ${ }^{8}$ Centre d'investigation clinique Epidémiologie Clinique, INSERM CIC 1432, Hôpital François-Mitterrand, Dijon, France
\end{abstract}

10.1136/lupus-2020-eurolupus. 19

Background Although diagnosis, treatment and prevention strategies of Systemic Lupus Erythematosus (SLE) have further improved during the last two decades, the net benefit in term of global SLE-related mortality remains unknown.

Objectives To describe the worldwide trends in SLE mortality between 2001 and 2014 using data of 84 available countries from the World Health Organization (WHO) mortality database. To modelize SLE-related deaths taking into account temporal changes, geographical localization and country wealth.

Methods We analyzed the WHO mortality database containing cause-specific mortality of participating countries. SLE-related deaths were identified using the international classification of disease 10 (ICD-10, code M32*). In all countries which provided data between 2001 and 2014, age-standardized mortality rate (ASMR) was calculated for each year using the WHO reference population database. To have a better understanding of mortality fluctuations, countries were grouped geographically by continents and their nominal gross domestic product (GDP) per capita were retrieved from the United Nation database. The association between gender, geographical areas and disease-specific mortality was analyzed using multivariate Poisson regression.

Results Between 2001 and 2014, a total of 83,639 SLE-related deaths occurred in 84 countries, which accounted for $0.02 \%$ of all deaths during the same period. Globally, the ASMR slightly increased between 2003 and 2014 (+0.58\%/year, $\mathrm{p}<0.01$ ), principally driven by an increase in Asia and Latin America $(2.87 \% / \mathrm{yr}$ and $+2.20 \% / \mathrm{yr}$, respectively; $\mathrm{p}<0.0001)$. During the 2001-2014 period, the ASMR significantly decreased in Europe, North America and Oceania (-1.43\%/yr, -3.49 and $-2.93 \%$, respectively; $\mathrm{p}<0.01$ for all). In 2014, the SLE ASMR of Europe (1.06 [95\%CI: 0.98-1.13] deaths/millions) was significantly lower than that of Latin America (5.53 [5.33 - 5.73], p<0.0001), North America (2.69 [2.53 - 2.84], $\mathrm{p}<0.0001)$, Asia (2.16 [2.03-2.29], $\mathrm{p}<0.0001)$ and Oceania (1.33 [0.97 - 1.70], $\mathrm{p}<0.01)$. Finally, we found a negative correlation between country wealth (GDP) and the SLE ASMR ( $\mathrm{r}=-0.394, \mathrm{p}<0.001)$.

Conclusion We observed drastic differences in the evolution of SLE-related mortality due to country geographic localization and country wealth.

\section{SAFETY OF CHLOROQUINE AND HYDROXYCHLOROQUINE DURING PREGNANCY: A SYSTEMATIC LITERATURE REVIEW AND META-ANALYSIS}

Thibaut Naveau, Olivier Lichau, Thomas Barnetche, Patrick Blanco, Marie-Elise Truchetet, Christophe Richez. Reference Centre For Rare Systemic Autoimmune Diseases RESO, Bordeaux University Hospital, Bordeaux, France

\subsection{6/lupus-2020-eurolupus.20}

Background Some countries face restrictions to prescribe Chloroquine (CQ) and Hydroxychloroquine (HCQ) during pregnancy, due to report of ocular toxicity in rodents and potential genotoxicity. The aim of this study was to perform a systematic literature review (SLR) and a meta-analysis of fetal malformation rates under CQ and HCQ (all indications) during pregnancy. In the subgroup of patients suffering from systemic lupus erythematosus (SLE), we performed an analysis of CQ and HCQ efficacy on maternofetal outcomes.

Methods Two independent reviewers searched literature from inception to September 2019 (via Pubmed, Embase and abstracts from ACR and EULAR congresses) for studies that compare fetal malformation rates and pregnancy outcomes of $\mathrm{CQ} / \mathrm{HCQ}$ versus placebo. A meta-analysis was performed to estimate a global risk difference for malformation rate and pregnancy outcomes, considering a p-value threshold of 5\%.

Results From 2835 articles, the literature search revealed 127 articles and abstracts of interest. For the fetal malformation safety analysis, we identified 16 studies fulfilling required criteria. Selected articles include a total of 2068 exposed children of mothers treated by CQ/HCQ for autoimmune diseases or malaria indication, compared to 16294 children in control group. The meta-analysis did not show any differences of malformation rate for patients treated by HCQ compared to nontreated ones $\left(\mathrm{DR}=0,00[-0.01,0.00]_{95 \%} ; \mathrm{p}=0.04, \mathrm{I}^{2}=2 \%\right)(\mathrm{fig}-$ ure 1).

Regarding pregnancy outcomes in SLE patients, we found 14 studies. The meta-analyses showed a protective effect of HCQ for disease flare $\left(\mathrm{OR}=0.48[0.27,0.87]_{95 \%} ; \mathrm{p}=0.02\right.$; $\left.\mathrm{I}^{2}=56 \%\right)$, prematurity $\left(\mathrm{OR}=0.61 \quad[0.40,0.93]_{95 \%} ; \mathrm{p}=0.02\right.$; $\left.\mathrm{I}^{2}=47 \%\right)$, and pre-eclampsia $\left(\mathrm{OR}=0.49 \quad[0.59,1.01]_{95 \%}\right.$; $\left.\mathrm{p}=0.05 ; \mathrm{I}^{2}=49 \%\right)$. The analyses did not reveal any significant difference for fetal growth restriction or congenital atrioventricular block. The SLR also identified uncontrolled cohort studies suggesting importance of HCQ in preventing lupus flares.

Conclusion Prenatal exposure to CQ or HCQ is not associated with higher rate of congenital abnormalities, and is associated with improvement of maternofetal outcomes. 